\title{
Uso de Internet del estudiantado de la Universidad Centroamericana, con enfoque en las redes sociales
}

Renata Rodrigues ${ }^{1}$, Amaru Canales Flores $^{2}$, Tatiana Peña ${ }^{2}$, Gabriela Castro² y Grettel Reyes Rodríguez $^{2}$

${ }^{1}$ Vice-rectora Académica, Universidad Centroamericana. Rotonda Rubén Darío 150 mts. al oeste. Apartado 69, Managua, Nicaragua. Correo electrónico: renata@ns.uca.edu.ni.

${ }^{2}$ Estudiantes de Licenciatura en Comunicación Social, Universidad Centroamericana, UCA

Recibido: agosto de 2009 / Aceptado: octubre de 2009

EL ARTÍCULO PRESENTA LOS RESULTADOS DE UN ESTUDIO SOBRE LOS USOS DE INTERNET que hace el estudiantado de la UCA, enfocándose en los hábitos de participación en las redes sociales existentes en línea. Fue realizado en el primer cuatrimestre de 2009 con un universo de aproximadamente siete mil estudiantes de las veinte carreras de Pregrado. Se aplicó una encuesta que indagaba sobre lugar y frecuencia de acceso a Internet, tipo de uso de la red, conocimiento y uso de las redes sociales y sus políticas de uso. Los principales resultados muestran que los estudiantes utilizan la Internet con mucha frecuencia y la principal actividad es la búsqueda de información. Otras actividades importantes están enfocadas a la comunicación con amigos a través del correo electrónico y el chat, y el entretenimiento a través de descarga de música, películas y juegos en línea. Dos terceras partes de los estudiantes participan como miembros de redes sociales, siendo Facebook y Hi5 las preferidas. La mayoría desconoce las políticas de uso de estas redes.

Palabras clave: hábitos uso Internet / redes sociales / TIC y educación

\section{Introducción}

La incorporación de las tecnologías en los procesos de enseñanza-aprendizaje constituye actualmente un paso importante en el desarrollo de capacidades individuales y colectivas de los estudiantes universitarios.

Desde hace algunos años la Universidad Centroamericana (UCA) ha destinado recursos económicos y humanos para promover el uso de la Internet, considerada una importante herramienta para satisfacer las necesidades de obtención y retroalimentación de información en los futuros profesionales. 
Considerando los procesos de enseñanza-aprendizaje llevados a cabo desde los planes de estudio de todas las carreras, resulta sumamente importante conocer el nivel de acceso de las y los estudiantes a tecnologías como la comunicación telefónica celular, las computadoras y la Internet, para el desarrollo de capacidades individuales y colectivas en los futuros profesionales.

Asimismo, recientemente se ha observado un incremento en la participación de las y los jóvenes en redes sociales disponibles en Internet, tales como Hi5, Facebook, Badoo, entre otras. Por lo tanto, es interesante conocer las motivaciones, el hábito de uso y las proyecciones de este tipo de redes en las y los estudiantes universitarios, así como el nivel de dominio que éstos tienen sobre las políticas de uso y el alcance que representa la participación en ellas.

Lapresenteinvestigación-realizadapor ungrupodeestudiantes delcurso "Cibercomunicación I", del primer cuatrimestre de 2009- determina el nivel de acceso y uso de la Internet que poseen las y los estudiantes de la UCA matriculados en el primer cuatrimestre del año 2009, así como la manera en que forman parte e interactúan en las denominadas redes sociales.

Finalmente, se proyecta que los resultados expuestos contribuyan a que las autoridades académicas tomen decisiones sobre nuevas políticas enfocadas a promover el uso de TIC en la educación universitaria. Asimismo, promover la reflexión académica sobre hábitos de uso de Internet entre investigadores y estudiantes mismos.

\section{Marco teórico}

Con el avance de la tecnología se han generado considerables cambios sociales y culturales que están siendo estudiados y analizados a nivel mundial. Se observa un incremento del uso de Internet no sólo en nuestro país, sino en el mundo entero. El informe anual 2008 de la Fundación Orange, sobre el desarrollo de la sociedad de la información en España, afirma que "en el año 2007 el número de internautas en el mundo se situó cerca de los 1.400 millones. El crecimiento de la penetración de Internet en los últimos seis años ha sido muy relevante. En África y Oriente Medio en dicho período el número de usuarios de Internet se ha multiplicado por cinco, en Latinoamérica y Europa Oriental se ha multiplicado por tres y en Asia Pacífico se ha duplicado. En Europa Occidental el incremento ha sido del 70\%, en Oceanía del 60\% y en Norteamérica del 40\%" (Fundación Orange, 2008). Según informe sobre el uso de Internet divulgado en la página Web de TELCOR, Nicaragua pasó de 15,559 conexiones de acceso a Internet en 2000, a 23,624 en 2006 (TELCOR, 2007). No obstante, vale destacar que el porcentaje de penetración de la Internet en Nicaragua es el más bajo de la región: 155,000 usuarios de Internet, representando a 2.6\% de la población, según datos divulgados en el sitio WEB Internetworldstats.com (Internet World Stats, 2009).

Este aumento también engloba el uso y las diferentes propuestas que ofrece la Internet a los usuarios, entre ellas las redes sociales, actividades de recreación y el comercio electrónico, por mencionar algunas. El impacto de las redes sociales en el mundo ha llamado la atención a diversos sectores. Un estudio realizado por Anderson Analytics (http://www. andersonanalytics.com/), empresa norteamericana de investigación de mercado, detalla que 75\% de la generación comprendida entre 15 y 29 años usa Myspace, mientras que el 65\% usa Facebook, el 14\% usa Twitter y un 9\% utiliza LinkedIn (Fayer Wayer, 2009). 
Tal como se evidencia en el informe de "La sociedad de la información en el mundo", elaborado por la Fundación Orange en el 2008, las tecnologías de la información se encuentran en constante transición debido a las prioridades y la evolución positiva de los servicios de comunicación. La Internet se ha convertido en una herramienta poderosa para las actividades básicas y necesarias de los individuos, como el comercio y el desarrollo de programas gubernamentales en países que apuestan por nuevos modelos electrónicos. Asimismo, la tecnología móvil -cada día más desarrollada- cuenta con un uso creciente, principalmente en países orientales como la India y China. Esto se debe al bajo costo de las operadoras multinacionales, la rapidez del servicio y la movilidad de estos equipos, convirtiendo al celular en una pieza indispensable para la comunicación local e internacional gracias a los mensajes de texto, la navegación en Internet y los procesadores de textos básicos, entre otros servicios ofrecidos por este aparato que está en constante evolución.

Pierre Levy clasifica esta novedad como "impactos de las nuevas tecnologías en la sociedad o en la cultura" (2007, p. 126). Esto quiere decir que las sociedades son blancos permanentes y modificadores activos, protagonistas de la inteligencia colectiva, uno de los principales motores de la cibercultura y que de igual forma crea el automantenimiento de las redes digitales, facilitado por un entorno propicio donde las tecnologías son acogidas.

Dentro de este orden encontramos en Internet diversas herramientas que facilitan la comunicación y el flujo de información, soportadas por tecnologías específicas como: el correo electrónico, la mensajería instantánea, los softwares especiales para desarrollar bases de datos, los blogs y las redes sociales, estas últimas famosas en el último lustro, principalmente en el continente americano. Países como Estados Unidos o Brasil han hecho de las redes sociales como Facebook y Orkut, herramientas básicas de comunicación, fundamentalmente populares entre los jóvenes.

Un reciente estudio sobre la privacidad de los datos personales y la seguridad de la información en las redes sociales online, realizado por el Instituto Nacional de Tecnologías de la Comunicación (INTECO) de España, muestra que en ese país la penetración de las diferentes redes sociales difiere por grupos de edad. Las redes de ocio son más utilizadas por los adolescente o jóvenes, en cambio las redes sociales de contenido profesional son mayormente utilizadas por personas adultas (INTECO, 2009, p. 43). Observaron también que el número de miembros crece vertiginosamente, principalmente entre los más jóvenes. Con relación a la privacidad de los datos hay riesgos reales, sin embargo no todos los usuarios de estas redes tienen conciencia de esto.

En este contexto, cobra relevancia conocer las características del uso de Internet en los estudiantes de la UCA. Esta investigación surge como resultado de las inquietudes planteadas por la encuesta sobre el uso de Internet en la comunidad estudiantil de la UCA, realizada en 2008 por los estudiantes del curso "Cibercomunicación I", bajo la coordinación de la docente Renata Rodrigues (2008). Entre los principales resultados de la encuesta de 2008 se observa un alto porcentaje de estudiantes con acceso frecuente a Internet, principalmente con propósito de comunicación e investigación académica. El 90.5\% de los estudiantes hace uso de Internet al menos una vez por semana. Hay un bajo índice de estudiantes con acceso a Internet desde su casa (36.5\%) y un porcentaje mediano de 
estudiantes poseen computadoras en su hogar (68\%). Se observan algunas diferencias de hábitos según carrera de estudio y año de ingreso en la universidad.

En el año 2008, el 43.7\% de los estudiantes que tenían conexión a Internet en su casa utilizaban Facebook y el 69\% HI5. En cambio, solamente 16\% de los estudiantes que no tenían Internet en su casa usaba Facebook, en contraste con el 55\% que no tenía conexión en su casa y usaba HI5.

A manera de conclusión, se encontró que:

El patrón de hábitos de uso de Internet que refleja esta investigación es muy semejante a los países en desarrollo. El correo electrónico y la búsqueda de información en Internet sobresalen, como en la mayoría de los países. Además, prevalece el entretenimiento y la participación en comunidades sociales virtuales. Podemos concluir que todavía dista mucho un aprovechamiento adecuado de todas las potencialidades educativas y comunicaciones que tiene Internet. El acceso es limitado y la gama de servicios utilizados también (Rodrigues, 2008, p. 8).

El equipo coordinador de la investigación se propuso repetir la encuesta en años sucesivos con el propósito de realizar una investigación longitudinal sobre los hábitos de uso de Internet de las y los estudiantes de la UCA.

\section{Objetivos de la investigación}

El objetivo de la investigación es conocer el nivel de acceso y uso que las y los estudiantes matriculados en el primer cuatrimestre de 2009 en la Universidad Centroamericana hacen de Internet.

Los objetivos específicos son:

- Identificar desde dónde los estudiantes acceden a Internet y con qué frecuencia.

- Determinar la relación existente entre la edad, sexo, carrera y año de ingreso de las y los estudiantes respecto al uso que le dan a la Internet.

- Identificar los principales recursos de Internet utilizados por los estudiantes para la comunicación, obtención de información y otros servicios.

- Explorar cuántos conocen sobre redes sociales presentes en Internet y hacen uso de ellas.

\section{Diseño metodológico}

La presente investigación tiene carácter descriptivo y fue utilizado un método cuantitativo para la recolecta de información. Se elaboró una encuesta, que fue aplicada entre abril y mayo de 2009. El universo o población de estudio fueron los estudiantes matriculados en todas las carreras de Pregrado de la Universidad Centroamericana, correspondientes al primer cuatrimestre del año 2009, siendo un total de 7,690.

El tipo de muestra fue aleatoria estratificada, es decir, segmentada por carreras, sexo y año de ingreso a la universidad. En total se realizaron 565 encuestas. La muestra se calculó con 
un nivel de confianza del 96\% y un margen de error muestral del +/- 4\%, y se distribuyó de manera proporcional al tamaño de la población de las carreras (ver anexo 1).

Cuando se definió la muestra por carrera, cada estudiante fue seleccionado de manera aleatoria según las listas oficiales. La mayoría de las encuestas fueron aplicadas a través de convocatoria a los estudiantes seleccionados. En los casos en que no se pudo encontrar a la persona, se seleccionó otra de la misma carrera, sexo y año de ingreso.

El cuestionario (ver anexo 2) se construyó en conjunto con las y los estudiantes de la asignatura de Cibercomunicación I, matriculados en el primer cuatrimestre de 2009, grupo a cargo de la Prof. Renata Rodrigues.

La encuesta se diseñó a base de preguntas cerradas, algunas de ellas daban la opción de respuestas múltiples. La pregunta general de investigación en la cual se centró la encuesta fue: ¿Para qué y cómo utilizan Internet los estudiantes de la Universidad Centroamericana? Además, se decidió agregar algunas preguntas específicas sobre el uso de redes sociales.

Cuadro 1. Variables de estudio de la investigación

\begin{tabular}{|l|l|}
\hline \multicolumn{1}{|c|}{ Variables de estudio } & \multicolumn{1}{|c|}{ Indicadores } \\
\hline $\begin{array}{l}\text { Características socio } \\
\text { demográficas }\end{array}$ & $\begin{array}{l}\text { Edad, sexo, carrera, ocupación, año de ingreso, tiene celular, tiene } \\
\text { computadora }\end{array}$ \\
\hline $\begin{array}{l}\text { Acceso y frecuencia } \\
\text { de uso de las TIC }\end{array}$ & $\begin{array}{l}\text { Posee teléfono celular } \\
\text { Posee computadora } \\
\text { Posee computadora con conexión a Internet } \\
\text { Cantidad de días por semana de uso de Internet } \\
\text { Lugar de uso de Internet }\end{array}$ \\
\hline $\begin{array}{l}\text { Uso de servicios y recursos } \\
\text { de Internet }\end{array}$ & $\begin{array}{l}\text { Obtención de información sobre: } \\
\text { Servicios de salud }\end{array}$ \\
& $\begin{array}{l}\text { Organismos civiles y gubernamentales } \\
\text { Investigación académica/estudio } \\
\text { Búsquedas de información en general }\end{array}$ \\
& Comunicación: \\
& Uso de chat \\
Correo electrónico \\
Foros de discusión \\
Participación en comunidades virtuales \\
Compras en línea \\
Banca en línea \\
Educación y aprendizaje \\
Servicios gubernamentales en línea \\
Actividades de entretenimiento \\
Uso del Entorno Virtual de Aprendizaje de la \\
Universidad
\end{tabular}




\section{Resultados y análisis}

\subsection{Características socio demográficas}

Las encuestas válidas suman 565 para un porcentaje de $61.9 \%$ de mujeres y $38.1 \%$ de hombres, lo que demuestra que hay más ingresos de mujeres a la universidad. En comparación, el año pasado (Rodrigues, 2008) los encuestados hombres fueron 35.2\% -un poco menos que en esta ocasión- y las mujeres fueron $64.8 \%$.

Con relación a la ocupación, observamos que el 77\% de los encuestados sólo estudia y que el $23 \%$ estudia y trabaja. Se puede afirmar que una gran cantidad del estudiantado de la UCA únicamente se dedica a estudiar.

El universo o población de estudio fueron los estudiantes de las 20 carreras de Pregado de la Universidad Centroamericana. En la carrera de Administración de Empresas fueron encuestados 121 (18.8\% del total de los encuestados), de la carrera de Arquitectura se encuestaron 20 (3.5\%), Diseño Gráfico 5 (1.1\%), Ingeniería Civil 11 (1.9\%), Ingeniería en Sistemas 34 (6\%), Ingeniería en Calidad Ambiental 11 (1.9\%), Ingeniería Industrial 42 (7.6\%), Contaduría Pública 61 (10.6\%), Economía 29 (5.1\%), Finanzas 11 (6.5\%), Gestión y Desarrollo del Turismo 19 (2.5\%), Marketing 21 (3.7\%), Derecho 75 (12.4\%), Comunicación Social 45 (7.8\%), Inglés 17 (2.5\%), Humanidades y Filosofía 3 (0.5\%), Psicología 22 (3.9\%), Sociología 8 (1.2\%), Trabajo Social y Gestión del Desarrollo $8(1.2 \%)$ y Ciencias Religiosas 2 $(0.2 \%)$, todos ellos estudiantes de la UCA.

Como observamos, la mayor cantidad de estudiantes encuestados (47.1\%) están matriculados en la Facultad de Ciencias Económicas y Empresariales. El segundo lugar lo ocupa la Facultad de Ciencia, Tecnología y Ambiente con 21.9\%, en tercer lugar la Facultad de Humanidades y Comunicación 18.6\%, y por último con el 12.4\% la Facultad de Ciencias Jurídicas.

La edad de la mayoría de los encuestados (85.6\%) oscila entre los 16 y 21 años. Mayoritariamente fueron consultados los estudiantes que ingresaron entre 2007 (21.2\%), $2008(23 \%)$ y $2009(34.5 \%)$, por lo que podemos afirmar que la población de estudiantes de la Universidad Centroamericana es mayormente joven, y se encuentra en los tres primeros años de la carrera. Los resultados obtenidos este año son similares al trabajo investigativo realizado por los estudiantes de Cibercomunicación I del año 2008, los jóvenes entre las edades de 16 y 21 años, representan a la mayoría (86\%).

\subsection{Datos básicos sobre uso y acceso}

Como se observa en la ilustración 1, el 92.6\% de los entrevistados posee un teléfono celular y solamente un 7.3\% no posee uno, en contraste con el $61.1 \%$ que posee computadora propia. Los resultados en esta variable revelan la necesidad de los jóvenes por el uso de las nuevas tecnologías, no sólo como herramienta de información, sino también de comunicación y de interacción. 


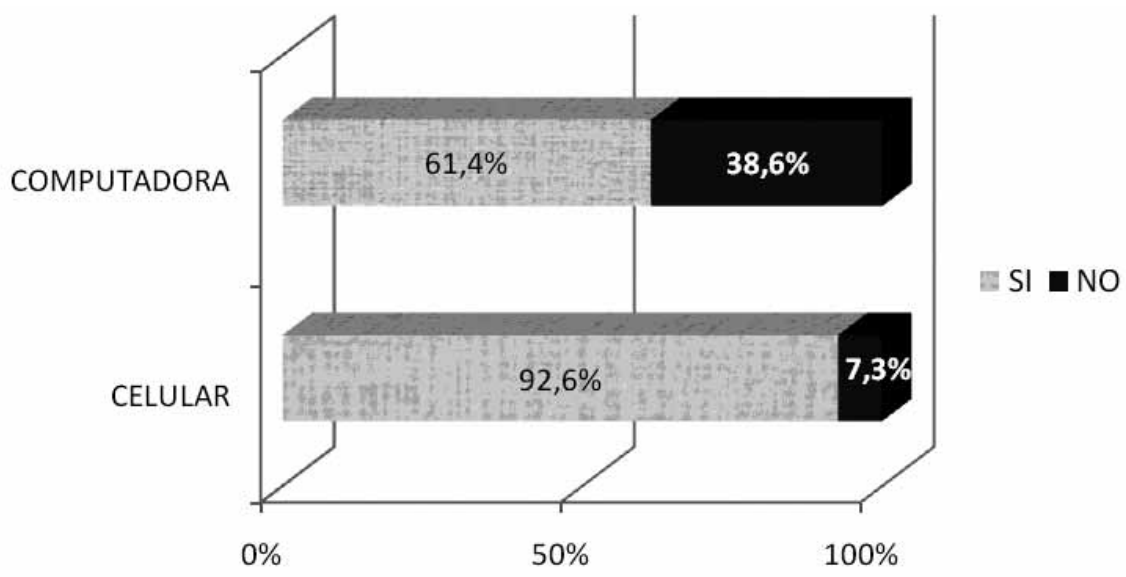

Ilustración 1. Tiene o no computadora y celular

Cabe destacar que los resultados obtenidos arrojan lo mismo que la investigación del 50 año pasado, donde el 92.8\% tenía celular. En cuanto al número de encuestados que tiene computadora propia, éste disminuyó en un 7\% en comparación al año pasado.

La Internet se encuentra entre las herramientas tecnológicas más utilizadas por las y los estudiantes de la Universidad Centroamericana. Al preguntar a los encuestados cuál era su frecuencia de uso de Internet, llaman la atención los resultados presentados en la ilustración 2 , pues casi la mitad (44\%) de los estudiantes contestaron al menos una vez al día, y un porcentaje cercano (31.5\%) lo hace al menos dos veces a la semana. Agrupando ambos datos, se encuentra que el 70\% de los encuestados utiliza Internet con mucha frecuencia.

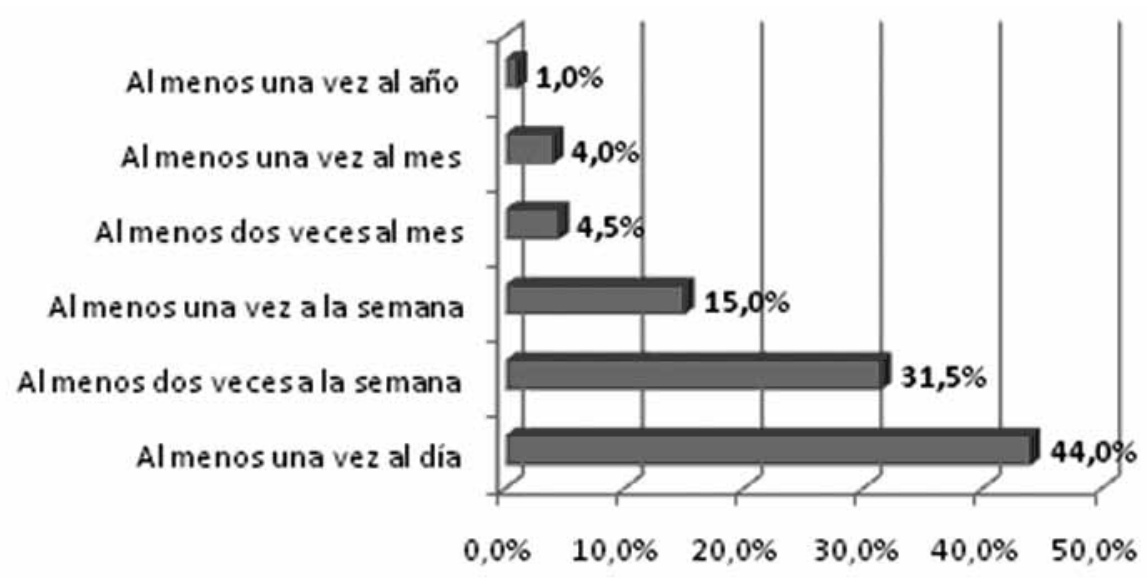

Ilustración 2. Frecuencia de uso de Internet

Sumando las tres primeras opciones marcadas por las y los encuestados, podemos afirmar que el $90.5 \%$ de los estudiantes hace uso al menos una vez por semana de Internet. Este dato es similar al obtenido en la encuesta del año 2008 (90.9\%). 
Los cibercafés (49.2\%) comprenden el lugar más utilizado por las y los estudiantes para acceder a Internet. Sin embargo, un porcentaje considerable (38.2\%) lo hace desde su propia casa, lo que representa un dato importante sobre el nivel de penetración de la Internet en los hogares nicaragüenses. La casa de amigos o familiares (18.8\%), el centro de trabajo $(11.9 \%)$ y la red inalámbrica de la UCA (5.8\%), constituyen otros sitios de acceso a Internet.

Cabe destacar que en comparación al año pasado, 10\% menos de los estudiantes accede a Internet desde los cibercafés, mientras que la cantidad de personas que lo hace desde la comodidad de su hogar aumentó en un 2.3\%.

\subsection{Uso del Entorno Virtual de Aprendizaje de la UCA}

El Entorno Virtual de Aprendizaje (EVA) de la UCA permite el intercambio de información y comunicación entre profesores y estudiantes de determinada asignatura a través de un espacio virtual que contiene múltiples herramientas y recursos. También facilita las condiciones para la implementación de los cursos virtuales.

Más de la mitad (66.7\%) de los estudiantes dijeron conocer el EVA. Sin embargo, 47.3\% afirmó no haberlo utilizado. Es importante destacar que en comparación con el año pasado, el entorno virtual es más conocido y utilizado en un $23.71 \%$ y $22.3 \%$ respectivamente. La Facultad que más utiliza el entorno virtual es la Facultad de Ciencia, Tecnología y Ambiente (66.9\%), seguida de la Facultad de Ciencias Económicas y Empresariales (45.9\%), la Facultad de Humanidades y Comunicación (45.9\%) y la Facultad de Ciencias Jurídicas (24.3\%).

Resulta interesante encontrar que las ingenierías ocupan los dos primeros lugares de las carreras cuyos estudiantes hacen más uso del entorno virtual. En este orden, destaca Ingeniería en Sistemas (97.1\%), Ingeniería Civil (72.7\%), seguidas de la carrera de Economía (69\%). Comunicación Social ocupa el puesto número seis, con 59.1\%.

Con respecto al año de ingreso, los estudiantes del plan 2005 (54.5\%) y los de 2009 (53.3\%) son quienes más utilizan el EVA. Este fenómeno puede responder quizás a la dinámica misma de los planes de estudio, donde las asignaturas sobre herramientas tecnológicas son impartidas en los primeros y últimos años de estudio.

\subsection{Usos que hacen de la Internet}

Como podemos observar en la ilustración 3, resulta positivo encontrar que un alto porcentaje $(96.6 \%)$ de las y los estudiantes utilizan la Internet para realizar investigaciones de carácter académico, un 20\% más en comparación al año pasado. 


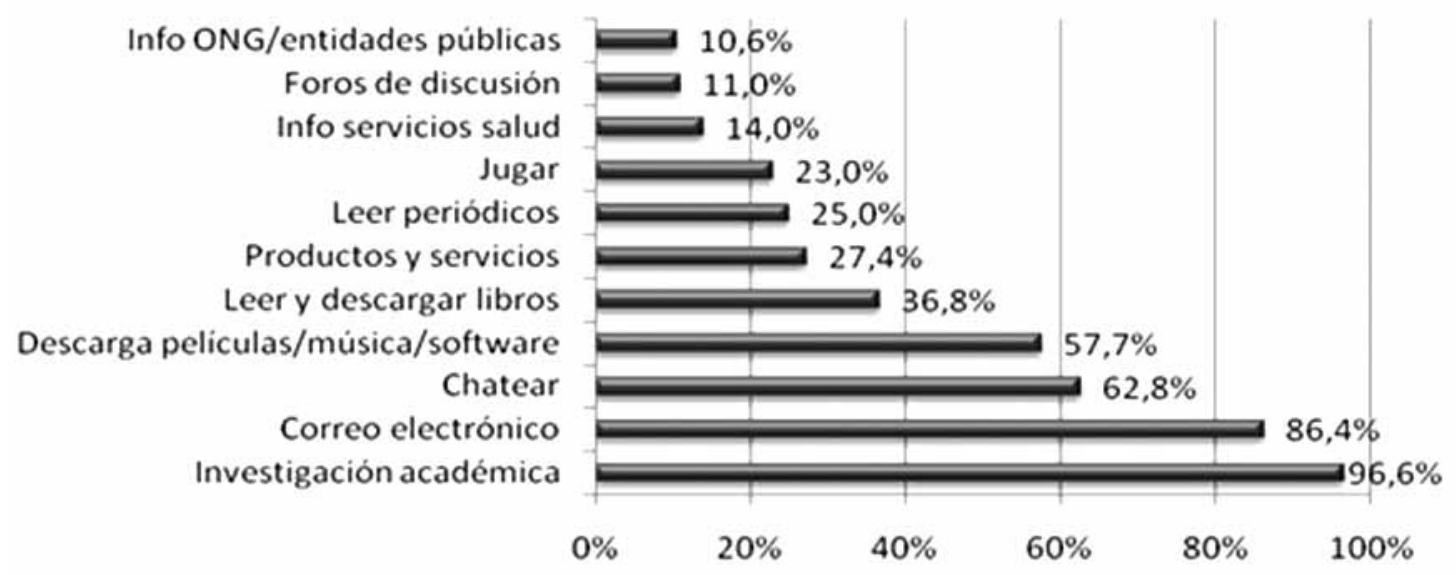

Ilustración 3. Utilización de la Internet

En cuanto a los espacios de comunicación e interacción con otros usuarios de Internet, el correo electrónico (86.4\%) y el chat (62.8\%) ocupan el segundo y tercer lugar respectivamente. Sin embargo, se observa que las y los usuarios que participan en los denominados foros de discusión se redujeron a la mitad (11\%). Las actividades de entretenimiento como la descarga de películas/música/software (57.7\%) y leer y descargar libros (36.8\%) se incrementaron en más de un $10 \%$.

Además, las y los estudiantes utilizan Internet para: consultas de productos y servicios $(27.4 \%)$, leer periódicos (25\%), jugar (22.9\%) y obtener información y servicios en salud $(14 \%)$. Las organizaciones no gubernamentales y las páginas Web de entidades públicas todavía no se ubican dentro de las fuentes de información prioritarias o servicios Web utilizados, pues son poco visitados por las y los estudiantes (10.6\%).

\subsection{Participación y actividades en redes sociales}

El 85\% de los encuestados pertenece a una o más redes sociales. El $68.8 \%$ dijo utilizar HI5 como red social, el 51.3\% Facebook, el 19.8\% Myspace y un 28.2\% afirma utilizar otras redes. 


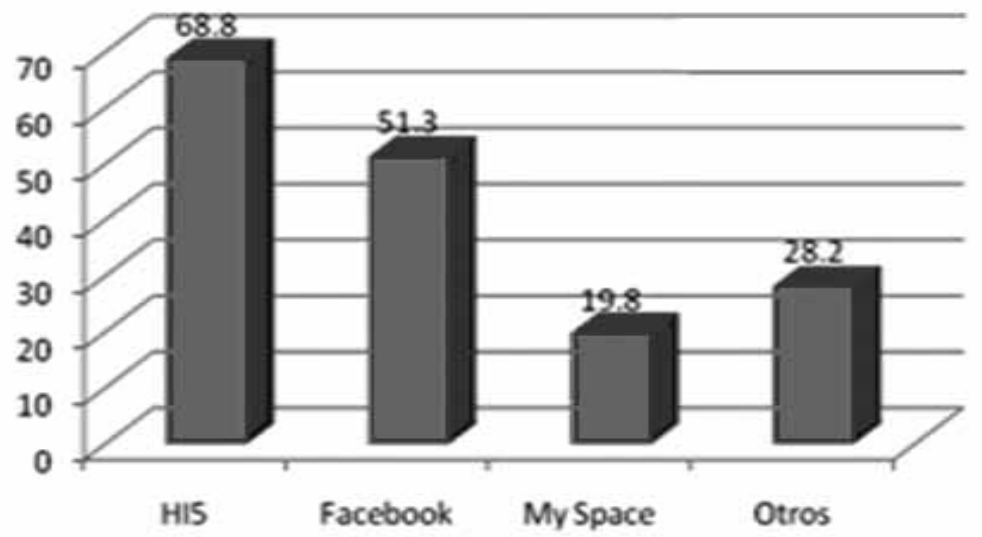

Ilustración 4. Participación en redes sociales

Podemos afirmar que las redes sociales más utilizadas entre los encuestados son HI5 y Facebook, observándose un aumento en el uso del último en comparación al resultado de la encuesta de 2008.

Según lo expresado por los encuestados, la actividad más realizada dentro de las redes sociales es ver fotos (67.3\%), seguido por escribir mensajes (64.8\%) y hacer nuevas amistades con 54.2\%. Publicar fotos se ubica en el 51.3\%, mientras que crear opinión aún parece no ser una de las principales actividades dentro de las redes, aunque el $46.9 \%$ dice utilizarlas para tal función. Ver videos es la actividad menos realizada, con un $29.4 \%$.

En cuanto a la frecuencia de acceso a las redes sociales, casi la mitad, o sea, el $45 \%$ de los encuestados dijo acceder de tres a seis veces por semana, mientras que un $19.6 \%$ accede diariamente, un 19.6\% accede esporádicamente y un 15.8\% dijo no acceder a una red social porque no está inscrito en ninguna.

Al preguntarles sobre la cantidad de amigos que tienen en sus redes sociales (ilustración 5), se observa que un poco más de $40 \%$ tiene más de 100 amigos. Se puede deducir la poca privacidad existente en sus redes sociales. 


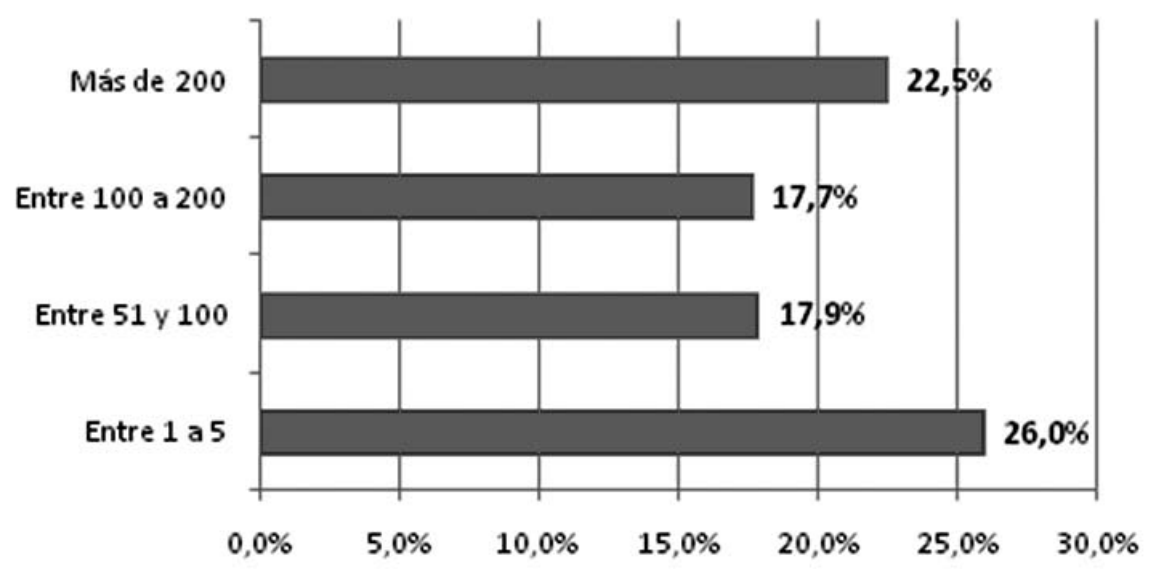

Ilustración 5. Amigos en la red social

Nos interesaba saber si los estudiantes conocen las políticas de uso de las redes sociales, 54 pues a través de ellas los usuarios son informados sobre el tipo de privacidad que pueden tener sus datos. Resulta interesante observar que el 50.3\% de los entrevistados afirma que conoce las políticas de uso de las redes sociales, en contraste al $35.9 \%$ que dice que no. Un 13.8 \% prefirió no responder.

\subsection{Diferencias en los comportamientos de los estudiantes, según algunas variables de estudio}

Al cruzar las variables "Edad" y "Utilidad de Internet", se encontró que en promedio las y los estudiantes entre 17 y 22 años son quienes acceden más a la red para realizar las distintas actividades que ésta ofrece.

Como se recordará, las investigaciones académicas (96.6\%), el uso de correo electrónico $(86.4 \%)$ y chatear (62.8\%), fueron las tres primeras opciones marcadas por las y los encuestados a la pregunta sobre para qué utilizan la Internet. Realizar investigaciones académicas no tiene distinción por carrera. Es decir, las y los estudiantes de las cuatro Facultades existentes en la UCA utilizan la Internet como herramienta a la hora de investigar sobre algún tema orientado en clases.

En el caso de la comunicación por chat, quienes más hacen uso de ella son los encuestados de 17 (64.8\%), 18 (66.3\%), 19 (69.1\%) y 20 (63.3\%) años. Sin embargo, cuando se habla de correo electrónico, los encuestados de 21 (96.4\%) y 22 (93.9\%) años ocupan las primeras posiciones.

$\mathrm{Al}$ analizar los cruces de "Edad" y "Actividad por Internet", se puede afirmar que las y los encuestados entre 17 y 20 años son quienes más se comunican a través del chat y el correo electrónico. Sin embargo, su nivel de participación en foros de discusión es mínimo o casi nulo, y son reemplazados por los estudiantes de 22 (33.3\%) y 23 (22.7\%) años. Asimismo, la lectura de periódicos nacionales e internacionales es de interés principalmente de los encuestados de 24 (75\%), $22(39.4 \%)$ y 21 (33.9\%) años. 
Un dato relevante es que los estudiantes de Sociología (57.1\%) y de Derecho (27.1\%) son quienes se dediquen más a obtener y recopilar información sobre organizaciones civiles e instituciones estatales. Quizás esto responda a la necesidad que la profesión exige de estar informado sobre temas como gobernabilidad, institucionalidad, ejercicio de derechos, entre otros.

Con respecto a la comunicación por chat, según nuestros datos estadísticos los estudiantes de Ingeniería Civil (81.8\%), Marketing (81\%), Comunicación Social (77.3\%), Ingeniería Industrial (74.4\%) e Ingeniería en Sistemas (73.5\%) ocupan las primeras posiciones porcentualmente.

El uso del correo electrónico es más o menos parejo en todas las carreras, aunque algunas sobresalen más que otras, según se puede observar en la ilustración 6 .

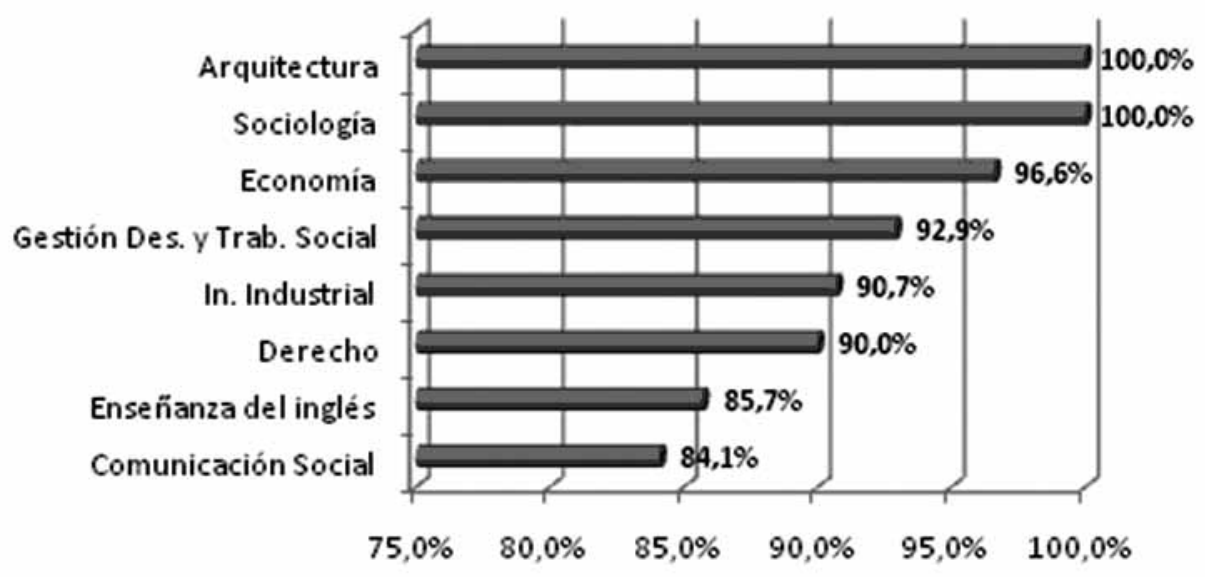

Ilustración 6. Uso de correo electrónico

En el análisis por carrera se encontró que la participación de los estudiantes en foros de discusión es mínima (11\%). Un promedio de tres de cada diez estudiantes de Ingeniería en Sistemas participa en un foro de discusión. Sin embargo, en carreras como Marketing o Gestión y Desarrollo del Turismo, la participación es nula.

Los estudiantes de Psicología son quienes más descargan libros por Internet (63.6\%), seguidos de los economistas (58.6\%) y los ingenieros en sistemas (55.9\%). Asimismo, son quienes más leen periódicos on line (57.1\%), junto a los comunicadores sociales (50\%) y los economistas (44.8\%).

\subsection{Lugar y frecuencia de acceso a Internet y uso de redes sociales}

Tal y como se planteó anteriormente, el 44\% de las y los encuestados accede a Internet al menos una vez al día. El 76.7\% de los que tienen conexión a Internet en su casa, el 73.1\% 
de los que usan Internet en sus trabajos y el 58\% de los que utilizan red inalámbrica, acceden diariamente. El 37.2\% de los estudiantes que afirman utilizar la Internet en los laboratorios de la UCA accede diariamente, y el 40\% accede por lo menos dos veces a la semana. Es natural que aquellos que tienen mayor facilidad de acceso (casa, trabajo o laptop) utilicen con mayor frecuencia la Internet, pero también vale la pena resaltar que los estudiantes que acceden desde los laboratorios de la UCA lo hacen con frecuencia muy alta. Aquellos que afirman acceder a la Internet desde un cibercafé o casas de amigos, lo hacen mayoritariamente dos veces por semana (respectivamente $44.2 \%$ y $36.8 \%$ ).

Al realizar el cruce de las variables para conocer con qué frecuencia y desde dónde acceden las y los encuestados a las redes sociales, se obtuvieron algunos datos interesantes para analizar.

Por ejemplo, el 45\% de los estudiantes que acceden a sus cuentas de redes sociales diariamente, lo hace desde la comodidad de su hogar. Sin embargo, quienes no tienen las posibilidades de acceder desde su casa, aprovechan cualquier oportunidad para hacerlo. En el caso de quienes ingresan entre una y tres veces por semana, utilizan principalmente los cibercafés (45.8\%), seguido de los laboratorios de la UCA (36.6\%), la red inalámbrica de la universidad (31.1\%) y la casa de amigos o familiares (35.5\%).

El 26.7\% de los estudiantes encuestados accede a las redes sociales por medio de las conexiones inalámbricas disponibles en sitios públicos, independientemente de la cantidad de veces que lo hagan a la semana.

$\mathrm{Al}$ cruzar las variables de las actividades que realizan las y los encuestados con el número de amigos que tienen en su red social, observamos que de las personas que poseen entre $1 \mathrm{y}$ 50 amigos, el 28.8\% tiene contacto directo con sus amigos a través de los mensajes.

Resulta interesante que las personas que poseen más de 200 amigos son los que crean más opinión (34.6\%), ven más fotos en las redes sociales (29.9\%), publican más fotos (35.6\%), ven videos (34.3\%) y son los que buscan hacer nuevas amistades (30.4\%). Según lo observado, se puede afirmar que son los que realizan más actividades en las redes sociales y acceden a ellas al menos una vez al día.

\subsection{Políticas de uso}

El 32.9\% que tiene más de 200 amigos en las redes sociales afirma conocer las políticas de uso de las mismas. Mientras que el $24.4 \%$ de aquellos que tienen entre 1 y 50 amigos no conoce las políticas de uso. Aparentemente, el uso frecuente de redes sociales fomenta 0 propicia el conocimiento de las políticas de privacidad y la cantidad de amigos. Entre más amigos tiene, está más familiarizado con las políticas de uso.

Los estudiantes de la Facultad de Ciencia, Tecnología y Ambiente son los que conocen más las políticas de uso (65.7\%), seguidos por los de la Facultad de Humanidades y Comunicación (63.2\%) y luego la Facultad de Ciencias Jurídicas (59.4\%) y la Facultad de Ciencias Económicas y Empresariales (52.5\%). 


\subsection{Frecuencia de uso y número de amigos}

El $57.5 \%$ de los y las encuestadas que poseen más de 200 amigos acceden a la Internet diariamente. Por otro lado, aquellos que acceden a la red con menor frecuencia son también los que tienen menos amigos. Esto quiere decir que la accesibilidad a la red permite agregar y tener más amigos en las redes sociales.

Los estudiantes que acceden diariamente a las redes sociales son de la Facultad de Ciencias Económicas y Empresariales, con el 45.9\%, la Facultad de Humanidades y Comunicación y la Facultad de Ciencia, Tecnología y Ambiente, ambas con 21.6\% y la Facultad de Ciencias Jurídicas con $10.8 \%$.

\subsection{Carreras y uso de las redes sociales}

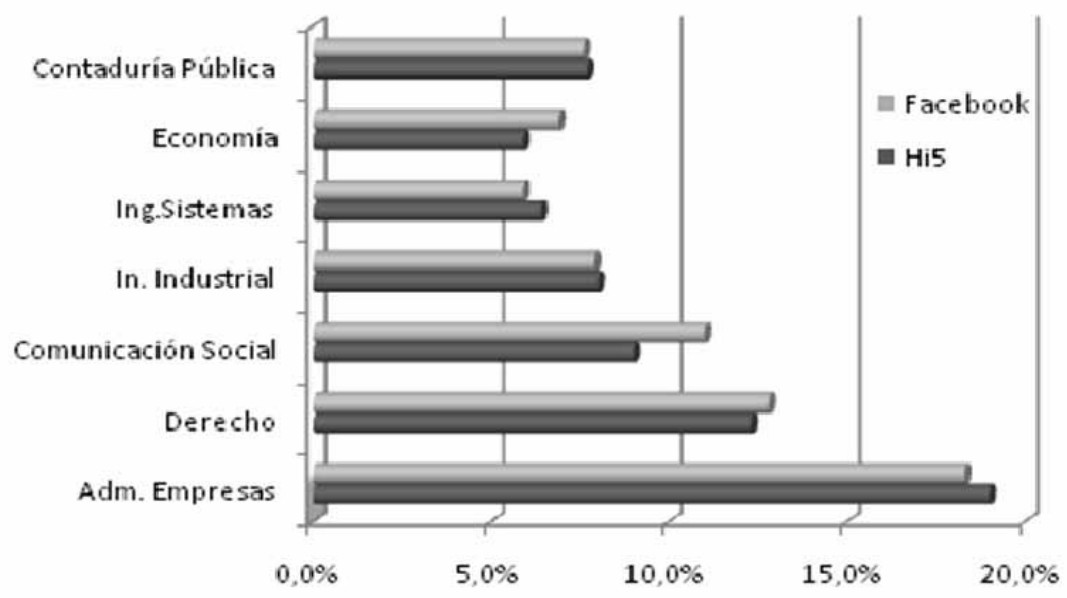

Ilustración 7. Uso de redes sociales por carrera

Como podemos observar en la ilustración 7, Hi5 es una de las redes sociales más utilizadas, con el 68.8\% del total de las y los encuestados. Facebook es la red social que ocupa el segundo puesto porcentualmente de las más utilizadas por los y las encuestadas. Las carreras de Administración de Empresas, Comunicación Social, Derecho, Ingeniería de Sistemas, Ingeniería Industrial, Contaduría Pública y Economía son las más destacadas en relación a la utilización de estas redes sociales.

Lugares de acceso y preferencia de redes sociales

Al cruzar la variable "uso de Internet desde su casa" con la variable "acceso y uso de las redes sociales", se observa que el 55.9\% del total de los encuestados que tienen conexión a la red desde su casa utiliza Facebook, el 50.9\% utiliza Myspace y el 40.6\% utiliza Hi5.

Sin embargo, encontramos que en el caso de las y los encuestados que acceden desde los laboratorios de la UCA, el 60.7\% del total utiliza Hi5, un poco más de la mitad (37.9\%) utiliza Facebook y sólo el 18.6\% utiliza Myspace. 
Podemos afirmar que hay una estrecha relación entre tener Internet en su casa y utilizar con más frecuencia las redes sociales, y observamos que las y los encuestados que poseen conexión en su hogar prefieren la red social Facebook.

\section{Conclusiones}

Por medio de la investigación podemos concluir que:

- Una gran cantidad de estudiantes (77\%) cursa su carrera dedicados solamente al estudio, mientras un porcentaje bastante menor (23\%) logra combinar sus actividades académicas con un empleo como fuente de ingreso económico.

- Las y los estudiantes de la UCA tienen -en un alto porcentaje- acceso a tecnologías de la comunicación como la telefonía móvil (92.6\%) y PC propia (61.1\%), lo que puede facilitar los procesos de aprendizaje para los futuros profesionales. Cabe destacar que casi la mitad (38.2\%) de las personas que cuentan con computadora en su propia casa posee conexión a Internet.

- La Internet es actualmente, para la mayoría de los estudiantes, una herramienta de uso constante, al punto que casi el 70\% de las y los encuestados accede al menos una vez al día a la red, y un 90.2\% lo hace al menos una vez a la semana aunque no posea computadora en su casa de habitación.

- El cibercafé es un lugar importante para acceder a la Internet, seguido de la casa de habitación y los laboratorios de la UCA.

- Los alumnos que ingresaron a la universidad en los años 2007 y 2008 son quienes utilizan Internet con mayor frecuencia.

- Casi todos los estudiantes utilizan Internet para realizar investigaciones académicas. El correo electrónico es el segundo servicio más utilizado. El acceso a los foros de discusión y la consulta a sitios Web institucionales son muy bajos.

- La participación en las redes sociales es relativamente alta y hay una tendencia de crecimiento. Sin embargo, su uso es limitado a muy pocos recursos y actividades. Se observa menor acceso y uso de las redes sociales en los alumnos encuestados que actualmente cursan el tercer y cuarto año de su carrera, lo que quizás responda al cambio de expectativas que estos espacios puedan producir, o a la mayor penetración de estos recursos entre los más jóvenes.

- Facebook y Hi5 son las redes sociales más utilizadas por los estudiantes encuestados. Varios de ellos son miembros de las dos redes. No se encontraron diferencias significativas -según las carreras- de preferencia de alguna red en particular.

- La mayoría de los estudiantes que usan redes sociales no conocen a profundidad las políticas de uso de éstas, a pesar de tener más de 200 amigos y/o formar parte de más de una red.

\section{Recomendación}

La Universidad Centroamericana debe promover la enseñanza adecuada del acceso a Internet para fines académicos, ya que la mayoría de los estudiantes la utilizan para buscar información para la realización de sus trabajos. Asimismo, debe promover otros recursos existentes en la Internet para fines educativos, tales como los foros de discusión, muy poco utilizados por los estudiantes. 
Se hace urgente una campaña de información con el objetivo de crear conciencia sobre el alcance que puede tener el uso de las redes sociales en la vida personal y profesional de las y los estudiantes, así como los riesgos para la privacidad que pueden traer.

Es necesario profundizar en la investigación del uso de las redes sociales en Nicaragua, considerando el nivel de incidencia que estos espacios pueden tener en la creación y fortalecimiento de relaciones humanas en las nuevas generaciones.

\section{Referencias bibliográficas}

Fayer Wayer. (2009). Uso de las redes sociales en diferentes generaciones. Recuperado el 13 de agosto de 2009, de http://www.fayerwayer.com/2009/08/uso-de-las-redes-sociales-endiferentes-generaciones/

Fundación Orange. (2008). España 2008. Informe Anual sobre el desarrollo de la sociedad de la información en España. Recuperado el 23 de enero de 2009, de http://www. fundacionorange.es/areas/25_publicaciones/e2008.pdf

Instituto Nacional de Tecnologías de la Comunicación (INTECO). (2009). Estudio sobre la privacidad de los datos personales y la seguridad de la información en las redes sociales online. Recuperado el 02 de mayo de 2009, de http://www.inteco.es/file/1000275028

Internet World Stats. (2009). Usage and population statistics. Recuperado el 05 de mayo de 2009, de http://www.internetworldstats.com/central.htm

Levy, P. (2007). Cibercultura. Barcelona: Anthropos Editorial.

Rodrigues, R. (2008). Acceso y uso de Internet por parte del estudiantado de la UCA. Manuscrito no publicado.

Telecomunicaciones y Correos (TELCOR). (2007). Conexiones del Servicio de Acceso a Internet. Recuperado el 05 de mayo de 2009, de http://www.telcor.gob.ni/Desplegar. asp?PAG_ID=50 
Anexo 1

\begin{tabular}{|c|c|c|c|c|c|c|c|c|c|c|c|c|c|c|c|c|c|c|c|}
\hline \multirow[b]{2}{*}{ Carrera } & \multicolumn{3}{|c|}{2004} & \multicolumn{3}{|c|}{2005} & \multicolumn{3}{|c|}{2006} & \multicolumn{3}{|c|}{2007} & \multicolumn{3}{|c|}{2008} & \multicolumn{3}{|c|}{2009} & \multirow{2}{*}{$\begin{array}{l}\text { Gran } \\
\text { Total }\end{array}$} \\
\hline & H & M & $\mathbf{T}$ & H & M & $\mathrm{T}$ & $\mathbf{H}$ & M & $\mathbf{T}$ & H & M & $\mathbf{T}$ & $\mathbf{H}$ & $\mathbf{M}$ & $\mathbf{T}$ & $\mathbf{H}$ & M & $\mathbf{T}$ & \\
\hline Arquitectura & 1 & 1 & 2 & 1 & 2 & 3 & 1 & 1 & 2 & 1 & 2 & 3 & 2 & 2 & 4 & 2 & 4 & 6 & 20 \\
\hline Diseño Gráfico & 0 & 0 & $\mathbf{0}$ & 0 & 0 & $\mathbf{0}$ & 0 & 0 & $\mathbf{0}$ & 0 & 0 & $\mathbf{0}$ & 0 & 0 & $\mathbf{0}$ & 2 & 3 & 5 & 5 \\
\hline Ing. Civil & 0 & 0 & $\mathbf{0}$ & 0 & 0 & $\mathbf{0}$ & 0 & 0 & $\mathbf{0}$ & 0 & 0 & $\mathbf{0}$ & 3 & 2 & 5 & 4 & 2 & 6 & 11 \\
\hline Ing. Sistemas & 1 & 0 & 1 & 2 & 1 & 3 & 3 & 2 & $\mathbf{5}$ & 5 & 3 & 8 & 5 & 2 & 7 & 6 & 4 & 10 & 34 \\
\hline Ing. Calidad Ambiental & 1 & 0 & 1 & 0 & 1 & 1 & 1 & 1 & 2 & 1 & 1 & 2 & 1 & 1 & 2 & 2 & 1 & $\mathbf{3}$ & 11 \\
\hline Ing. Industrial & 1 & 1 & 2 & 2 & 1 & 3 & 3 & 3 & 6 & 6 & 4 & 10 & 6 & 4 & 10 & 6 & 5 & 11 & 42 \\
\hline Admon. de Empresas & 2 & 6 & 8 & 1 & 8 & 9 & 7 & 14 & 21 & 7 & 14 & 21 & 9 & 15 & 24 & 21 & 8 & 29 & 112 \\
\hline Contaduría & 1 & 2 & 3 & 2 & 3 & 5 & 2 & 5 & 7 & 4 & 8 & 12 & 5 & 9 & 14 & 7 & 13 & 20 & 61 \\
\hline Economía & 1 & 0 & 1 & 1 & 1 & 2 & 2 & 4 & 6 & 2 & 4 & 6 & 3 & 4 & 7 & 3 & 4 & 7 & 29 \\
\hline Finanzas & 0 & 0 & $\mathbf{0}$ & 0 & 0 & $\mathbf{0}$ & 0 & 0 & $\mathbf{0}$ & 0 & 0 & $\mathbf{0}$ & 2 & 4 & 6 & 1 & 4 & $\mathbf{5}$ & 11 \\
\hline $\begin{array}{l}\text { Gestión y Desarrollo } \\
\text { del Turismo }\end{array}$ & 0 & 1 & 1 & 0 & 1 & 1 & 0 & 2 & 2 & 1 & 4 & 5 & 1 & 4 & $\mathbf{5}$ & 1 & 4 & $\mathbf{5}$ & 19 \\
\hline Marketing & 0 & 0 & $\mathbf{0}$ & 0 & 0 & 0 & 0 & 0 & $\mathbf{0}$ & 1 & 1 & 2 & 2 & 6 & 8 & 3 & 9 & 12 & 21 \\
\hline Derecho & 2 & 5 & 7 & 3 & 6 & 9 & 5 & 7 & 12 & 4 & 11 & 15 & 4 & 10 & 14 & 7 & 11 & 18 & 75 \\
\hline Comunicación Social & 1 & 2 & 3 & 1 & 3 & 4 & 2 & 5 & 7 & 2 & 9 & 12 & 3 & 8 & 11 & 2 & 6 & 8 & 45 \\
\hline Inglés & 0 & 1 & 1 & 0 & 1 & 1 & 1 & 1 & 2 & 1 & 3 & 4 & 1 & 2 & 3 & 2 & 4 & 6 & 17 \\
\hline $\begin{array}{l}\text { Humanidades y } \\
\text { Filosofía }\end{array}$ & 0 & 0 & $\mathbf{0}$ & 0 & 0 & $\mathbf{0}$ & 0 & 0 & $\mathbf{0}$ & 1 & 0 & 1 & 1 & 0 & 1 & 0 & 1 & 1 & 3 \\
\hline Psicología & 3 & 3 & 3 & 0 & 3 & 3 & 0 & 3 & 3 & 1 & 3 & 4 & 1 & 3 & 4 & 1 & 4 & 5 & 22 \\
\hline Sociología & 0 & 1 & 1 & 0 & 0 & 0 & 0 & 1 & 1 & 0 & 0 & $\mathbf{0}$ & 1 & 1 & 2 & 3 & 1 & 4 & 8 \\
\hline $\begin{array}{l}\text { Trabajo Social y } \\
\text { Gestión del Desarrollo }\end{array}$ & 0 & 0 & $\mathbf{0}$ & 0 & 0 & $\mathbf{0}$ & 0 & 0 & $\mathbf{0}$ & 0 & 1 & 1 & 1 & 2 & $\mathbf{3}$ & 1 & 3 & 4 & 8 \\
\hline $\begin{array}{l}\text { Técnico en Ciencias } \\
\text { Religiosas }\end{array}$ & 0 & 0 & $\mathbf{0}$ & 0 & 0 & $\mathbf{0}$ & 0 & 0 & $\mathbf{0}$ & 0 & 1 & 1 & 0 & 1 & 1 & 0 & 0 & $\mathbf{0}$ & 2 \\
\hline
\end{tabular}




\section{Anexo 2}

\section{Universidad Centroamericana}

Encuesta sobre hábitos de uso de

Internet del estudiantado de la UCA

\begin{tabular}{|l|l|}
\hline \multicolumn{2}{|c|}{ Sexo } \\
\hline Hombre & \\
\hline Mujer & \\
\hline
\end{tabular}
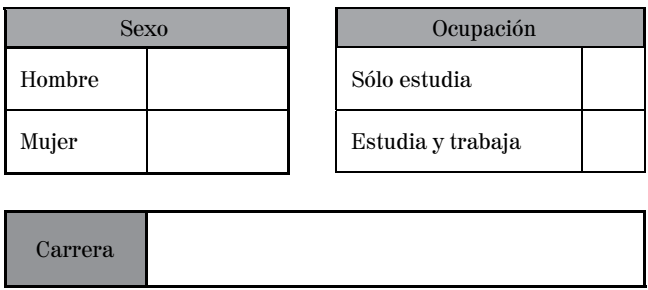
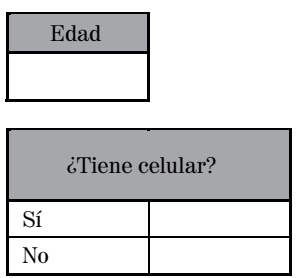

¿Tiene computadora propia?

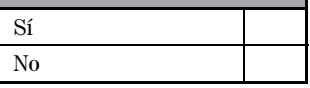

¿Con qué frecuencia utilizas Internet?

Al menos una vez al día

$\mathrm{Al}$ menos dos veces a la semana

Al menos una vez a la semana

$\mathrm{Al}$ menos dos veces al mes

$\mathrm{Al}$ menos una vez al mes

Nunca

¿Cuáles son los dos lugares más frecuentes en los que utiliza Internet?

\begin{tabular}{|l|l|}
\hline Casa & \\
\hline Trabajo & \\
\hline Laboratorios UCA & \\
\hline Red Inalámbrica UCA & \\
\hline Red Inalámbrica pública & \\
\hline Cibercafé & \\
\hline Casa amistad/familiar & \\
\hline
\end{tabular}

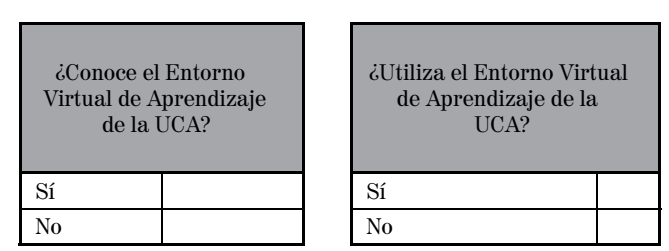

NOTA: Esta encuesta es realizada por estudiantes del curso Cibercomunicación I - IC2009, de la carrera de Comunicación Social.

Muchas gracias por su cooperación.

\begin{tabular}{|l|l|}
\hline \multicolumn{2}{|c|}{ ¿PUEDE qué utilizas Internet? } \\
\hline Obtener información sobre: & \\
\hline Para investigación académica/estudio & \\
\hline Sobre productos y servicios & \\
\hline Relacionada con salud o servicios de salud & \\
\hline Organizaciones civiles / autoridades públicas & \\
\hline Comunicación a través de: & \\
\hline Chat & \\
\hline Correo electrónico & \\
\hline Foros de discusión & \\
\hline Actividades de entretenimiento como: & \\
\hline Jugar & \\
\hline Bajar películas/música/software & \\
\hline Leer / descargar libros electrónicos & \\
\hline Leer periódicos & \\
\hline
\end{tabular}

\begin{tabular}{|l|l|l|}
\hline \multicolumn{3}{|c|}{ Sobre las Redes Sociales: } \\
\hline Redes Sociales & $\begin{array}{l}\text { Señale las que } \\
\text { conoce }\end{array}$ & $\begin{array}{l}\text { Señale si está inscrito en } \\
\text { esta red }\end{array}$ \\
\hline HI 5 & & \\
\hline Facebook & & \\
\hline MySpace & & \\
\hline Netjoven & & \\
\hline Badoo & & \\
\hline Otra & & \\
\hline
\end{tabular}

Si está inscrito en una Red Social, conteste las preguntas que siguen:

\begin{tabular}{|l|l|}
\hline \multicolumn{2}{|l|}{ Actividades en la red social (varias opciones) } \\
\hline Escribir mensajes & \\
\hline Crear opinión-comentarios & \\
\hline Ver fotos & \\
\hline Publicar fotos & \\
\hline Ver videos & \\
\hline Hacer nuevas amistades & \\
\hline
\end{tabular}

\begin{tabular}{|c|c|}
\hline Con qué frecuencia accedes a tu r & \\
\hline Diariamente & \\
\hline Entre 6 a 4 veces por semana & \\
\hline Entre 3 a 1 vez por semana & \\
\hline Esporádicamente & \\
\hline Cuántos amigos/as tenés en tu Re & \\
\hline Entre 1 a 50 & \\
\hline Entre 51 a 100 & \\
\hline Entre 100 a 200 & \\
\hline Más de 200 & \\
\hline Conoce las políticas de uso de las & SI \\
\hline redes sociales & NO \\
\hline
\end{tabular}

